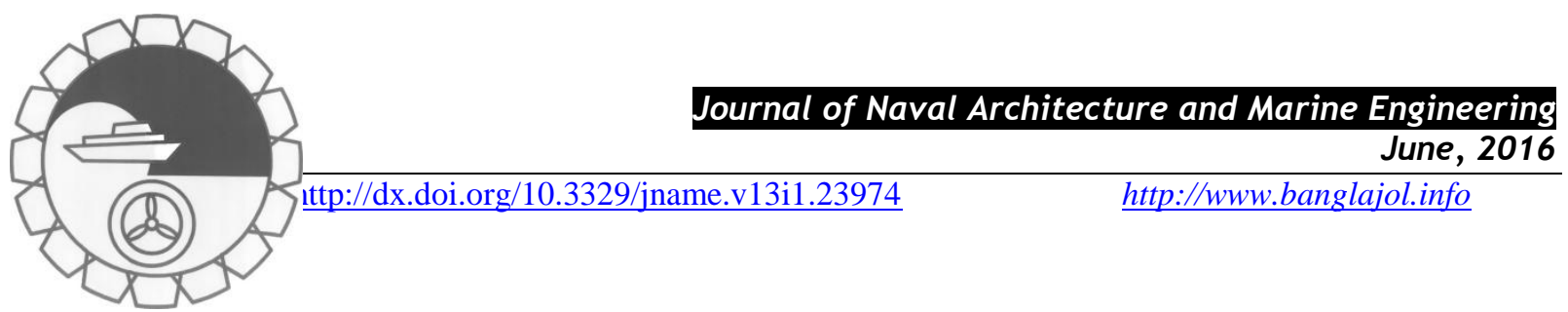

\title{
SORET AND DUFOUR EFFECTS ON MHD HEAT AND MASS TRANSFER FLOW OF A MICROPOLAR FLUID WITH THERMOPHORESIS PARTICLE DEPOSITION
}

\section{P. Sudarsana Reddy ${ }^{*}$, Ali J. Chamkha ${ }^{2}$}

${ }^{1}$ Department of Mathematics, RGM College of Eng. \& Tech, Nandyal-518501, AP, India, E-mail: suda1983@ gmail.com

${ }^{2}$ Mechanical Engineering Department, Prince Mohammad Bin Fahd University, Al-Khobar 31952, Kingdom of Saudi Arabia, E-mail: achamkha@pmu.edu.sa

\begin{abstract}
:
The effect of thermophoresis on heat and mass transfer flow of a micropolar fluid in the presence of Soret and Dufour effects over a stretching sheet is investigated in the present analysis. The transformed conservation equations are solved numerically using an optimized, extensively validated, variational Finite element method. The influence of key non-dimensional parameters, namely, suction parameter $V_{0}(0.1$ - 0.5), magnetic parameter $M$ (0.1-1.5), Soret parameter $\mathrm{Sr}$ (0.5 - 1.8), Dufour parameter $\mathrm{Du}(0.5-0.1)$ and thermophoretic parameter $\tau(0.1-1.5)$ on velocity, angular velocity (micro-rotation), temperature and concentration fields as well as skin-friction coefficient, Nusselt number and Sherwood number are examined in detail and the results are shown in graphically and in tabular form to know the physical importance of the problem. It is found that the imposition of wall fluid suction $\left(V_{0}>0\right)$ in the present problem of flow has the effect of depreciating the velocity, microrotation, temperature and concentration boundary layer thicknesses at every finite value of $\eta$. The thermal boundary layer thickness decelerates whereas solutal boundary layer thickness is improved with the combined influence of Sr and Du.
\end{abstract}

Keywords:Micropolar fluid, Soret effect, Dufour effect, thermophoresis,finite element method

\section{NOMENCLATURE}

$\mathrm{B}_{1} \quad$ Coupling constant parameter

$\mathrm{Cp} \quad$ Specific heat at constant pressure

$\mathrm{C}_{\mathrm{w}} \quad$ Uniform constant concentration

K Permeability parameter

$\mathrm{D}_{\mathrm{m}} \quad$ Mass diffusion coefficient

$\mathrm{E}_{\mathrm{c}} \quad$ Eckert Number

$\mathrm{G}_{\mathrm{r}} \quad$ Thermal Grashof number

$\mathrm{G}^{*} \quad$ Micropolar parameter

$\mathrm{k}_{\mathrm{p}} \quad$ Permeability of the porous medium

$\mathrm{Nu}$ Nusselt number

Sc Schmidt number

St Shear stress

$\mathrm{Re} \quad$ Local Reynolds number

$\mathrm{T}_{\mathrm{m}} \quad$ Mean fluid temperature(K)

$\mathrm{T}_{\infty} \quad$ Free stream temperature

$\mathrm{V}_{1} \quad$ Dimensionless suction velocity $(\mathrm{m} / \mathrm{s})$

$\mathrm{u}$ Velocity in the $\mathrm{x}$-direction $(\mathrm{m} / \mathrm{s})$

W Microrotation (angular velocity) $(\mathrm{m} / \mathrm{s})$

\section{Greek symbols}

$\alpha$ Thermal diffusivity

$\beta$ Coefficient of thermal expansion

$\beta^{\prime}$ Coefficient of volume expansion

$\gamma \quad$ Spin gradient

$\theta \quad$ Non-dimensional temperature parameter

$\tau$ Thermophoretic parameter

$\kappa$ Thermal conductivity $\left(\mathrm{Wm}^{-1} \mathrm{~K}\right)$
C Concentration of the species

$\mathrm{c}_{\mathrm{s}} \quad$ Concentration susceptibility

$\mathrm{C}_{\infty} \quad$ Free stream concentration

M Magnetic parameter

$\mathrm{Du}$ Dufour number

$\mathrm{G}$ Acceleration due to gravity

$\mathrm{G}_{\mathrm{m}}$ Solutal Grashof number

J Microinertia density

$\mathrm{K}_{\mathrm{t}} \quad$ Thermal diffusion ratio

Pr Prandtl number

Sh Sherwood number

$\mathrm{Sr}$ Soret number

$\mathrm{T}$ Temperature of the fluid(K)

$\mathrm{T}_{\mathrm{w}} \quad$ Uniform temperature(K)

$\mathrm{U}_{0}$ Velocity at the wall $(\mathrm{m} / \mathrm{s})$

$\mathrm{V}_{\mathrm{t}}$ Thermophoretic velocity

$\mathrm{v} \quad$ Velocity in y-direction $(\mathrm{m} / \mathrm{s}$ )

$\lambda \quad$ Micropolar parameter

$v \quad$ Kinematic viscosity $\left(\mathrm{m}^{2} \mathrm{~s}^{-1}\right)$

$\rho \quad$ Density of the fluid $\left(\mathrm{kgm}^{-3}\right)$

$\sigma \quad$ Electrical conductivity

$\eta \quad$ Similarity variable

$\varphi \quad$ Non-dimensional concentration parameter

$\omega$ Non-dimensional micro rotation parameter 


\section{Introduction}

The theory of micropolar fluids has been an active area of research for several decades, because of its wide range of applications in analyzing fluid flow in brain, exotic lubricants, blood flow in animals, etc. Micropolar fluids are fluids with microstructure and related to the fluids with non-symmetrical stress tensor. They represent fluids consisting of rigid, randomly oriented or spherical particles suspended in a viscous medium, where the deformation of fluids particles is ignored (e.g. polymeric suspensions, animal blood, liquid crystals). The theory of micropolar fluids was originally formulated by Eringen (1966) by taking the local effects arising from the microstructure and the intrinsic motion of the fluid into the account. Micropolar fluids are non-Newtonian fluids consisting of dumb-bell molecules, colloidal fluids, liquid crystals, lubricants, suspension fluids, animal blood, etc. In this theory, the continuum is regarded as sets of structured particles which contain not only mass and velocity, but also a substructure. That is, each material volume element contains microvolume elements that can translate and rotate independently of the motion of microvolume. In this model, two independent kinematic vector fields are introduced — one representing the translation velocities of fluid particles; and the other representing angular (spin) velocities of the particles, called as microrotation vector. The more detail of this theory and its applications can be found in Ariman et al.(1973, 1974) and books by Lukaszewicz (1999) and Eringen (2001). Chamkha et al. (2010,2013) has presented unsteady MHD micropolar fluid past a heated vertical plate by considering Joule heating, radiation and chemical reaction effects. Bhargava et al. (2003) have presented heat and mass transfer characteristics of micropolar fluid over porous stretching sheet. Ibrahim et al. (2008) analyzed unsteady MHD mixed convection flow of a micropolar fluid by taking viscous dissipation and thermal radiation into the account. Damseh Rebhi et al. (2009) have noticed the influence of heat generation/absorption and first-order chemical reaction on micropolar fluid flow over a uniform stretching sheet. Pratap Kumar et al. (2010) have presented free convection flow of a micropolar fluid over a vertical plate. Yacos et al. (2011) have reported boundary layer heat transfer stagnation-point flow of micropolar fluid over a stretching/shrinking sheet.Rosali et al. (2012) analyzed micropolar fluid flow through porous media over a stretching/shrinking sheet with suction. Abd El-Aziz (2013) studied boundary layer flow and heat transfer analysis of a micropolar fluid over a stretching sheet with viscous dissipation and found that thermal boundary layer thickness decelerates with the higher values of micropolar parameter. Mahmood et al. (2013) have perceived boundary layer flow, heat transfer analysis of micropolar second grade fluid over a stretching sheet. Recently, Bala Siddulu Malga (2014) has analyzed the unsteady heat and mass transfer flow of a micropolar fluid over a vertical plate with thermal radiation and heat generation.

Thermophoresis is a phenomenon by which submicron sized particles suspended in a nonisothermal gas acquire a velocity relative to the gas in the direction of decreasing temperature. The velocity acquired by the particle is known as the thermophoretic velocity and the force experienced by the suspended particles due to the temperature gradient is called the thermophoretic force. The magnitudes of the thermophoretic force and velocity are proportional to the temperature gradient. Thermophoresis has many applications in radioactive particle deposition in nuclear reactors, deposition of silicon thin films, particles impacting the blade surface of gas turbines and aerosol technology. Many authors have done good work by taking thermophoresis in the account. Goren (1977) was first to analyze the thermophoresis in laminar flow over a flat plate for cold and hot plate conditions. Chamkha and Pop $(2004,2011 \mathrm{a})$ analyzed the natural convection over a vertical flat plate in a porous medium with thermophoresis. Seddeek (2005) studied numerically the effect of variable viscosity and thermophoresis on a boundary layer flow with chemical reaction. Partha $(2008,2009)$ analyzed the effects of Soret and Dufour with thermophoresis in a non-Darcy porous medium, later he extended the problem by taking suction/injection in to the account.

The effects Thermo-diffusion and Diffusion-thermo are very significant for the fluids which have higher temperature and concentration gradients. The Thermo-Diffusion (Soret) effect is corresponds to species differentiation developing in an initial homogeneous mixture submitted to a thermal gradient and the Diffusionthermo (Dufour) effect corresponds to the heat flux produced by a concentration gradient. Usually, in heat and mass transfer problems the variation of density with temperature and concentration give rise to a combined buoyancy force under natural convection and hence the temperature and concentration will influence the diffusion and energy of the species. Long years back, Chapman and Cowling (1952) and Hirshfelder et al. (1954) has been studied the effect of Thermo- diffusion and Diffusion-thermo of heat and mass transfer in the kinematic theory of gas. Alam et al. (2006a) have studied the Dufour and Soret effects on steady free convection and mass transfer flow past a semi-infinite vertical porous plate in a porous medium. Alam et al. (2006b) have Soret and Dufour effects on MHD heat and mass transfer flow of a micropolar fluid with thermophoresis particle deposition 
investigated, the effect of suction on mixed convective flow along vertical plate by taking Soret and Dufour effects. Dulal Pal et al. (2011) has studied MHD non-Darcian mixed convection heat and mass transfer over a non-linear stretching sheet with Soret and Dufour effects and chemical reaction. MHD mixed convection flow with Soret and Dufour effects past a vertical plate embedded in porous medium was studied by Makinde (2011). Chamkha et al. (2011b) have presented the impact of Soret and Dufour effects on unsteady natural convective flow over a vertical cylinder with chemical reaction. Reddy et al. (2012) has presented finite element solution to the heat and mass transfer flow past a cylindrical annulus with Soret and Dufour effects. Raju et al. (2014) have analyzed natural convection flow of Non-Newtonian fluid through porous medium by taking Soret effect into the consideration.

To the author knowledge no studies have been found in literature to analyze the combined influence of Soret and Dufour effects on convective heat and mass transfer flow of a viscous electrically conducting micropolar fluid over a stretching sheet in the presence of suction/injection and thermophoresis particle deposition. Hence, this problem is examined in this paper. The problem addressed in this article has many practical applications, such as, technological and manufacturing engineering, radioactive deposition of silicon thin films and polymer deposition of silicon thin films and polymer extrusion process. The equation of non-linear momentum, microrotation, temperature and concentration along with the boundary conditions are solved by using Finite element method with Mathematica 10.0 software.

\section{Mathematical Formulation}

We consider two-dimensional, study, mixed convective heat and mass transfer flow of a viscous electrically conducting, micropolar fluid through porous medium over a stretching sheet in the presence of thermophoresis, Soret and Dufour effects. The coordinate system is such that $x$-axis is taken along the stretching surface in the direction of the motion with the slot at origin, and the $y$-axis is perpendicular to the surface of the sheet as shown schematically in Fig. 1. A uniform transverse magnetic field $\left(B_{0}\right)$ is applied along the $y$-axis. The stretching surface and the fluid are maintained same temperature and concentration initially, instantaneously they raised to a temperature $T_{w}\left(>T_{\infty}\right)$ and concentration $C_{w}\left(>C_{\infty}\right)$ which remain unchanged. The effects of thermophoresis are being taken in the diffusion equation to help in the understanding of the mass deposition variation on the surface. The temperature gradient in the y-direction is much larger than that in $x$-direction and hence thermophoretic velocity component normal to the surface is of more importance. Under the above stated physical situations, the governing boundary-layer and Darcy-Boussinesq's approximations, the basic equations are given by:

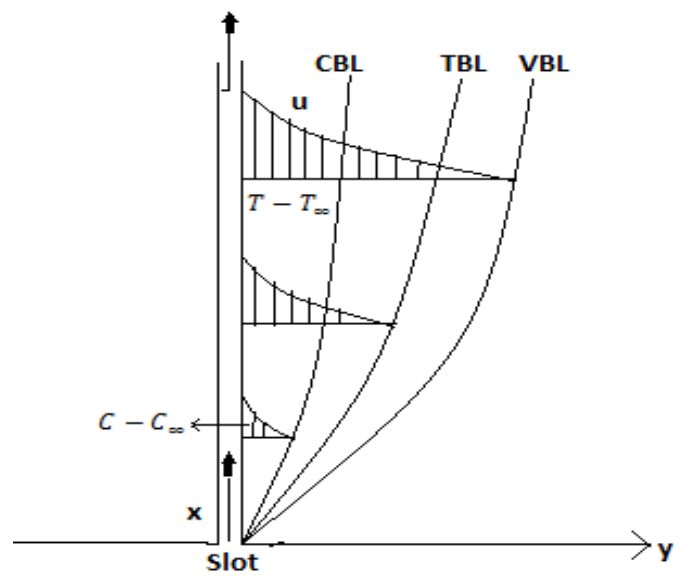

Fig. 1: Flow configuration and coordinate system.

$\frac{\partial u}{\partial x}+\frac{\partial v}{\partial y}=0$

$u \frac{\partial u}{\partial x}+v \frac{\partial u}{\partial y}=v \frac{\partial^{2} u}{\partial y^{2}}+k_{1} \frac{\partial W}{\partial y}+g_{a} \beta\left(T-T_{\infty}\right)+g_{a} \beta^{\prime}\left(C-C_{\infty}\right)-\frac{v}{k_{p}} u+\frac{\sigma B_{0}^{2}}{\rho} u$

$u \frac{\partial W}{\partial x}+v \frac{\partial W}{\partial y}=-\frac{\kappa}{\rho J}\left(2 W+\frac{\partial u}{\partial y}\right)+\frac{\gamma}{\rho J} \frac{\partial^{2} W}{\partial y^{2}}$

$u \frac{\partial T}{\partial x}+v \frac{\partial T}{\partial y}=\alpha \frac{\partial^{2} T}{\partial y^{2}}+\frac{v}{c_{p}}\left(\frac{\partial u}{\partial y}\right)^{2}+\frac{D_{m} k_{T}}{c_{s} c_{p}} \frac{\partial^{2} C}{\partial y^{2}}$ 
P. S. Reddy, A. J. Chamkha/ Journal of Naval Architecture and Marine Engineering 13(2015) 39-50

$u \frac{\partial C}{\partial x}+v \frac{\partial C}{\partial y}=D_{m} \frac{\partial^{2} C}{\partial y^{2}}+\frac{D_{m} k_{T}}{T_{m}} \frac{\partial^{2} T}{\partial y^{2}}-\frac{\partial}{\partial y}\left(V_{T} C\right)$

The associated boundary conditions on the vertical surface are defined as follows,

$$
\begin{gathered}
u=U_{w}(x)=a x, \quad v=V_{1}(x), \quad T=T_{w}, \quad C=C_{w}, W=-s\left(\frac{\partial u}{\partial y}\right), \quad \text { at } y=0 . \\
u \rightarrow 0, \quad v \rightarrow 0, \quad T \rightarrow T_{\infty}, \quad C \rightarrow C_{\infty}, W \rightarrow 0, \quad \text { at } y \rightarrow \infty .(6)
\end{gathered}
$$

In the above equations $x$ and $y$ represents coordinate axis along the continuous surface in the direction of motion and perpendicular to it, $u$ and $v$ are the velocity components along $x$ and $\mathrm{y}$ directions, respectively. The term $V_{1}=-\sqrt{\frac{v U_{w}}{2 x}} V_{0}$ represents the mass transfer at the surface with $\mathrm{V}_{1}<0$ for suction and $\mathrm{V}_{1}>0$ for injection.

The effect of thermophoresis is usually prescribed by means of an average velocity acquired by small particles to the gas velocity when exposed to a temperature gradient. In boundary layer flow, the temperature gradient in $y$-direction is very much larger than in the $x$-direction and therefore only the thermophoretic velocity in $y$ direction is considered. As a consequence, the thermophoretic velocity $\mathrm{V}_{\mathrm{T}}$, which appears in Eq.(5), is expressed as

$$
V_{T}=-\frac{K_{1} v}{T_{r}} \frac{\partial T}{\partial y}
$$

where $K_{1}$ is the thermophoretic coefficient and $T_{r}$ is the reference temperature. A thermophoretic parameter $\tau$ is given by the relation

$$
\tau=-\frac{K_{1}\left(T_{w}-T_{\infty}\right)}{T_{r}}
$$

Where the typical values of $\tau$ are $0.01,0.1$ and 1.0 corresponding to approximate values of $-k_{I}\left(T_{w}-T_{\infty}\right)$ equal to $3,30,300 \mathrm{~K}$ for a reference temperature of $T=300 \mathrm{~K}$.

The mathematical analysis of the problem is simplified by introducing the following dimension less functions $f$, $h, \theta, \varphi$ and the similarity variable $\eta$

$$
\begin{aligned}
& \eta=y \sqrt{\frac{a}{2 v}}, \quad u=U_{w} \frac{d f}{d \eta}, \quad v=-\sqrt{\frac{v U_{w}}{2 x}} f(\eta)+\frac{U_{w} y}{2 x} \frac{d f}{d \eta}, \\
& W=-\sqrt{\frac{U_{w}}{2 v x}} U_{w} h, \quad \theta(\eta)=\frac{T-T_{\infty}}{T_{w}-T_{\infty}}, \quad \varphi(\eta)=\frac{C-C_{\infty}}{C_{w}-C_{\infty}} .
\end{aligned}
$$

The continuity equation (1) is satisfied by the stream function $\psi(x, y)$ defined by

$$
u=\frac{\partial \psi}{\partial y}, \quad v=-\frac{\partial \psi}{\partial x}
$$

Substituting Eqn. (9) in Eqns. (2) to (6), we obtain

$$
\begin{aligned}
& f^{\prime \prime \prime}+B_{1} h^{\prime}+f f^{\prime \prime}+G_{r} \theta+G_{m} \varphi-K f^{\prime}-M f^{\prime}=0 \\
& \lambda h^{\prime \prime}-2 \frac{\lambda}{G_{1}}\left(2 h+f^{\prime \prime}\right)+f^{\prime} h+f h^{\prime}=0 \\
& \theta^{\prime \prime}+\operatorname{Pr} f \theta^{\prime}+\operatorname{Pr} \operatorname{Du} \varphi^{\prime \prime}+\operatorname{Pr} E c\left(f^{\prime \prime}\right)^{2}=0 \\
& \varphi^{\prime \prime}+\operatorname{Sc} f \varphi^{\prime}+\operatorname{ScSr} \theta^{\prime \prime}-\tau\left(\theta^{\prime} \varphi^{\prime}+\theta^{\prime \prime} \varphi\right)=0
\end{aligned}
$$

Where $\lambda=\frac{\gamma}{\rho v \mathrm{~J}}$ and $G_{1}=\frac{\gamma a}{v \kappa}$ are micropolar parameters, $G_{r}=\frac{2 g_{a} \beta x}{U_{w}{ }^{2}}\left(T_{w}-T_{\infty}\right)$ is local Grashof number, $G_{m}=\frac{2 g_{a} \beta^{\prime} x}{U_{w}{ }^{2}}\left(C_{w}-C_{\infty}\right)$ is local modified Grashof number , $B_{1}=\frac{\kappa_{1}}{v}$ is the Coupling constant parameter, $K=\frac{U_{w} k_{p}}{2 v x} \quad$ is the permeability parameter, $\operatorname{Pr}=\frac{v}{\alpha} \quad$ is the Prandtl number, $S c=\frac{v}{D_{m}}$ is the Schmidt number, $D u=\frac{D_{m} K_{T}}{c_{s} c_{p} v} \frac{\left(C_{w}-C_{\infty}\right)}{\left(T_{w}-T_{\infty}\right)}$ is the Dufour number, $S r=\frac{D_{m} K_{T}}{v T_{m}} \frac{\left(T_{w}-T_{\infty}\right)}{\left(C_{w}-C_{\infty}\right)}$ is the Soret number, $E c=\frac{U^{2}{ }_{w}}{c_{p}} \frac{1}{\left(T_{w}-T_{\infty}\right)}$ is the Eckert number, $v=\frac{\mu+\kappa}{\rho}$ is the kinematic viscosity, $k_{1}=\frac{\kappa}{\rho}\left(k_{1}>0\right)$ is the coupling constant,

$M=\frac{2 \sigma B_{0}^{2} x}{\rho U_{w}}$ is the magnetic parameter.

The corresponding transformed boundary conditions are

$$
\begin{array}{lr}
f^{\prime}=1, f=V_{0}, h=-s f^{\prime \prime}, \theta=1, \varphi=1, & \text { at } y=0 \\
f^{\prime}=0, h=0, \theta=0, \varphi=0 & \text { at } y \rightarrow \infty
\end{array}
$$

Where $f(0)=V_{0}$ with $V_{0}<0$ and $V_{0}>0$ corresponding to injection and suction respectively. 
The major physical quantities of interest in this problem are the local skin friction coefficient $\left(C_{f}\right)$, the local Nusselt number $\left(N u_{x}\right)$ and the local Sherwood number $\left(S h_{x}\right)$ are defined, respectively, by

$$
C_{f}=\frac{f^{\prime \prime}(0)}{R e_{x}^{\frac{1}{2}}}, N u_{x}=-\frac{\theta^{\prime}(0)}{R e_{x}^{\frac{1}{2}}}, S h_{x}=-\frac{\varphi^{\prime}(0)}{R e_{x}^{\frac{1}{2}}} \text {. }
$$

\section{Method of Solution}

\subsection{Finite Element Method}

The Finite- element method (FEM) has been implemented to obtain numerical solutions of coupled non-linear equations (11) to (14) of third-order in $f$ and second order in $h, \theta, \varphi$ under boundary conditions (15). This technique is extremely efficient and allows robust solutions of complex coupled, nonlinear multiple degree differential equation systems. The details of finite-element method can find in Bhargava et al. (2009), Anwar Beg et al. (2008) and Rana et al. (2012). The steps involved in this method are as follows:

(i) Finite-element discretization

In the finite element discretization the entire interval is divided into a finite number of subintervals and this subinterval is called an element. The set of all these elements is called the finite-element mesh.

(ii) Generation of the element equations

a. Variational formulation of the mathematical model over the typical element (an element from the mesh) is performed.

b. An approximate solution of the variational problem is assumed, and the element equations are made by substituting this solution in the above system.

c. Using interpolating polynomials the stiffness matrix is constructed.

(iii) Assembly of element equations

By imposing inter element continuity conditions all the algebraic equations are assembled. This result a large number of algebraic equations called global finite-element model and it represents the whole domain.

(iv) Imposition of boundary conditions

The boundary conditions which represent the flow model are imposed on the assembled equations.

(v) Solution of assembled equations

The assembled equations so obtained can be solved by any of the numerical techniques, namely, the Gauss elimination method, LU decomposition method, etc. An important consideration is that of the shape functions which are employed to approximate actual functions.

For the solution of system of non-linear ordinary differential equation (11) - (14) together with boundary conditions (15), first we assume that

$\frac{\mathrm{df}}{\mathrm{d} \eta}=\mathrm{j}$

The equations (11) to (14) then reduces to

$j^{\prime \prime}+B_{1} h^{\prime}+f j^{\prime}+G_{r} \theta+G_{m} \varphi-(K+M) j=0$

$\lambda h^{\prime \prime}-2 \frac{\lambda}{G_{1}}\left(2 h+j^{\prime}\right)+j h+f h^{\prime}=0$

$\theta^{\prime \prime}+\operatorname{Pr} f \theta^{\prime}+\operatorname{Pr} D u \varphi^{\prime \prime}+\operatorname{Pr} E c\left(j^{\prime}\right)^{2}=0$

$\varphi^{\prime \prime}+\operatorname{Scf} \varphi^{\prime}+\operatorname{ScS} \operatorname{Sr} \theta^{\prime \prime}-\tau\left(\theta^{\prime} \varphi^{\prime}+\theta^{\prime \prime} \varphi\right)=0$

The boundary conditions take the form

$j=1, f=V_{0}, h=-s j^{\prime}, \theta=1, \varphi=1, \quad$ as $y=0$

$j=0, h=0, \theta=0, \varphi=0, \quad$ as $y \rightarrow \infty$

\subsection{Variational formulation}

The variational form associated with Eqs. (17) to (21) over a typical linear element $\left(\eta_{e}, \eta_{e+1}\right)$ is given by

$\int_{\eta_{e}}^{\eta_{e+1}} w_{1}\left(\frac{\mathrm{df}}{\mathrm{d} \eta}-\mathrm{j}\right) \mathrm{d \eta}=0$

$\int_{\eta_{e}}^{\eta_{e+1}} w_{2}\left(j^{\prime \prime}+B_{1} h^{\prime}+f j^{\prime}+G_{r} \theta+G_{m} \varphi-(K+M) j\right) \mathrm{d} \eta=0$

$\int_{\eta_{e}}^{\eta_{e+1}} w_{3}\left(\lambda h^{\prime \prime}-2 \frac{\lambda}{G_{1}}\left(2 h+j^{\prime}\right)+j h+f h^{\prime}\right) \mathrm{d} \eta=0$

$\int_{\eta_{e+1}}^{\eta_{e+1}} w_{4}\left(\theta^{\prime \prime}+\operatorname{Pr} f \theta^{\prime}+\operatorname{Pr} D u \varphi^{\prime \prime}+\operatorname{Pr} E c\left(j^{\prime}\right)^{2}\right) \mathrm{d} \eta=0$

$\int_{\eta_{e}}^{\eta_{e+1}} w_{5}\left(\varphi^{\prime \prime}+\operatorname{Sc} f \varphi^{\prime}+\operatorname{Sc} \operatorname{Sr} \theta^{\prime \prime}-\tau\left(\theta^{\prime} \varphi^{\prime}+\theta^{\prime \prime} \varphi\right)\right) \mathrm{d} \eta=0$

Where $w_{1}, w_{2}, w_{3}, w_{4}$ and $w_{5}$ are arbitrary test functions and may be viewed as the variations in $f, h, \theta$, and $\phi$, respectively. 


\subsection{Finite- element formulation}

The finite-element model may be obtained from above equations by substituting finite-element approximations of the form

$f=\sum_{j=0}^{2} f_{j} \Psi_{j}, j=\sum_{j=0}^{2} j_{j} \Psi_{j}, \quad h=\sum_{j=0}^{2} h_{j} \Psi_{j}, \theta=\sum_{j=0}^{2} \theta_{j} \Psi_{j}, \quad \phi=\sum_{j=0}^{2} \phi_{j} \Psi_{j}$

With $w_{1}=w_{2}=w_{3}=w_{4}=w_{5}=\Psi_{i}, \quad(i=1,2)$.

Where $\Psi_{i}$ are the shape functions for a typical element $\left(\eta_{e}, \eta_{e+1}\right)$ and are defined as

$\Psi_{1}^{e}=\frac{\left(\eta_{e+1}-\eta\right)}{\left(\eta_{e+1}-\eta_{e}\right)}, \Psi_{2}^{e}=\frac{\left(\eta-\eta_{e}\right)}{\left(\eta_{e+1}-\eta_{e}\right)}, \quad \eta_{e} \leq \eta \leq \eta_{e+1}$.

The finite element model of the equations thus formed is given by

$$
\left[\begin{array}{ccc}
{\left[K^{11}\right]} & {\left[K^{12}\right]\left[K^{13}\right]} & {\left[K^{14}\right]\left[K^{15}\right]} \\
{\left[K^{21}\right]} & {\left[K^{22}\right]\left[K^{23}\right]} & {\left[K^{24}\right]\left[K^{25}\right]} \\
{\left[K^{31}\right]} & {\left[K^{32}\right]\left[K^{33}\right]} & {\left[K^{34}\right]\left[K^{35}\right]} \\
{\left[K^{41}\right]} & {\left[K^{42}\right]\left[K^{43}\right]} & {\left[K^{44}\right]\left[K^{45}\right]} \\
{\left[K^{51}\right]\left[K^{52}\right]\left[K^{53}\right]\left[K^{54}\right]\left[K^{55}\right]}
\end{array}\right]\left[\begin{array}{c}
f \\
j \\
h \\
\theta \\
\phi
\end{array}\right]=\left[\begin{array}{l}
\left\{r^{1}\right\} \\
\left\{r^{2}\right\} \\
\left\{r^{3}\right\} \\
\left\{r^{4}\right\} \\
\left\{r^{5}\right\}
\end{array}\right]
$$

Where $\left[K^{m n}\right]$ and $\left[r^{m}\right](m, n=1,2,3,4,5)$ are defined as

$$
\begin{aligned}
& K_{i j}{ }^{11}=\int_{\eta_{e}}^{\eta_{e+1}} \psi_{i} \frac{\partial \psi_{j}}{\partial \eta} \mathrm{d} \eta, K_{i j}{ }^{12}=-\int_{\eta_{e}}^{\eta_{e+1}} \psi_{i} \psi_{j} \mathrm{~d} \eta, K_{i j}{ }^{13}=K_{i j}{ }^{14}=K_{i j}{ }^{15}=0 . \\
& K_{i j}{ }^{21}=\int_{\eta_{e}}^{\eta_{e+1}} \psi_{i} \frac{\partial \psi_{j}}{\partial \eta} \mathrm{d} \eta, K_{i j}{ }^{22}=-\int_{\eta_{e}}^{\eta_{e+1}} \frac{\partial \psi_{i}}{\partial \eta} \frac{\partial \psi_{j}}{\partial \eta} \mathrm{d} \eta+(M+K) \int_{\eta_{e}}^{\eta_{e+1}} \psi_{i} \psi_{j} \mathrm{~d} \eta, \\
& K_{i j}{ }^{23}=B_{1} \int_{\eta_{e}}^{\eta_{e+1}} \psi_{i} \frac{\partial \psi_{j}}{\partial \eta} \mathrm{d} \eta, K_{i j}{ }^{24}=G_{r} \int_{\eta_{e}}^{\eta_{e+1}} \psi_{i} \psi_{j} \mathrm{~d} \eta, K_{i j}{ }^{25}=G_{m} \int_{\eta_{e}}^{\eta_{e+1}} \psi_{i} \psi_{j} \mathrm{~d} \eta . \\
& K_{i j}{ }^{31}=\int_{\eta_{e}}^{\eta_{e+1}} \psi_{i} \frac{\partial \psi_{j}}{\partial \eta} \mathrm{d} \eta, K_{i j}{ }^{32}=2 \frac{\lambda}{G_{1}} \int_{\eta_{e}}^{\eta_{e+1}} \psi_{i} \frac{\partial \psi_{j}}{\partial \eta} \mathrm{d} \eta+\int_{\eta_{e}}^{\eta_{e+1}} \psi_{i} \psi_{j} \mathrm{~d} \eta \text {. } \\
& K_{i j}{ }^{33}=\lambda \int_{\eta_{e}}^{\eta_{e+1}} \psi_{i} \frac{\partial \psi_{j}}{\partial \eta} \mathrm{d} \eta+4 \frac{\lambda}{G_{1}} \int_{\eta_{e}}^{\eta_{e+1}} \psi_{i} \psi_{j} \mathrm{~d} \eta, K_{i j}{ }^{34}=K_{i j}{ }^{35}=0 . \\
& K_{i j}^{41}=\operatorname{Pr} \int_{\eta_{e}}^{\eta_{e+1}} \psi_{i} \frac{\partial \psi_{j}}{\partial \eta} \mathrm{d} \eta, K_{i j}{ }^{42}=\operatorname{Pr} . E c \int_{\eta_{e}}^{\eta_{e+1}} \psi_{i} \frac{\partial \psi_{j}}{\partial \eta} \frac{\partial \psi_{j}}{\partial \eta} \mathrm{d} \eta, K_{i j}{ }^{43}=0 \text {, } \\
& K_{i j}{ }^{44}=\int_{\eta_{e}}^{\eta_{e}+1} \psi_{i} \bar{\theta} \frac{\partial \psi_{j}}{\partial \eta} \mathrm{d} \eta, K_{i j}{ }^{45}=\operatorname{Pr} D u \int_{\eta_{e}}^{\eta_{e+1}} \psi_{i} \bar{\phi} \frac{\partial \psi_{j}}{\partial \eta} \mathrm{d} \eta, \\
& { }^{51}=S c \int_{\eta_{e}}^{\eta_{e+1}} \psi_{i} \bar{\phi} \psi_{j} \mathrm{~d} \eta, K_{i j}{ }^{52}=0, K_{i j}{ }^{53}=0, \\
& K_{i j}{ }^{54}=\operatorname{ScSr} \int_{\eta_{e}}^{\eta_{e+1}} \psi_{i} \bar{\theta} \frac{\partial \psi_{j}}{\partial \eta} \mathrm{d} \eta-\tau \int_{\eta_{e}}^{\eta_{e+1}} \psi_{i} \bar{\theta} \frac{\partial \psi_{j}}{\partial \eta} \psi_{j} \mathrm{~d} \eta K_{i j}{ }^{55}=\int_{\eta_{e}}^{\eta_{e+1}} \frac{\partial \psi_{i}}{\partial \eta} \frac{\partial \psi_{j}}{\partial \eta} \mathrm{d} \eta-\tau \int_{\eta_{e}}^{\eta_{e+1}} \psi_{i} \frac{\partial \psi_{j}}{\partial \eta} \mathrm{d} \eta . \\
& r_{i}^{2}=0, r_{i}^{2}=-\left(\psi_{i} \frac{d \Psi_{i}}{d \eta}\right)_{\eta_{e}}^{\eta_{e+1}}, r_{i}^{3}=-\left(\psi_{i} \frac{d \Psi_{i}}{d \eta}\right)_{\eta_{e}}^{\eta_{e+1}}, r_{i}^{4}=-\left(\psi_{i} \frac{d \Psi_{i}}{d \eta}\right)_{\eta_{e}}^{\eta_{e+1}}, r_{i}^{5}=-\left(\psi_{i} \frac{d \Psi_{i}}{d \eta}\right)_{\eta_{e}}^{\eta_{e+1}}
\end{aligned}
$$

Where

$\bar{f}=\sum_{j=0}^{2} f_{i} \frac{\partial \Psi_{i}}{\partial \eta}, \quad \bar{j}=\sum_{j=0}^{2} j_{i} \frac{\partial \Psi_{i}}{\partial \eta}, \bar{h}=\sum_{j=0}^{2} h_{i} \frac{\partial \Psi_{i}}{\partial \eta}, \bar{\theta}=\sum_{j=0}^{2} \theta_{i} \frac{\partial \Psi_{i}}{\partial \eta}, \quad \bar{\phi}=\sum_{j=0}^{2} \phi_{i} \frac{\partial \Psi_{i}}{\partial \eta}$.

The very important aspect in this numerical procedure is to select an approximate finite value of $\eta_{\infty}$. So, in order to estimate the relevant value of $\eta_{\infty}$, the solution process has been started with an initial value of $\eta_{\infty}=4$, and then Equations (23) - (27) are solved together with associated boundary conditions. We have updated the value of $\eta_{\infty}$ and the solution process is continued until the results are not affected with further values of $\eta_{\infty}$. The choice of $\eta_{\max }=4$ for velocity and temperature and $\eta_{\max }=6$ for concentration have confirmed that all the numerical solutions approach to the asymptotic values at the free stream conditions.

\section{Grid Independence Test}

To investigate the sensitivity of the solutions to mesh density, we have performed the grid invariance test for velocity, temperature and concentration distributions and are shown in table 1. It is observed from this table that in the same domain the accuracy is not affected, even the number of elements increased, by decreasing the size of the elements.

\section{Results and Discussion}

Comprehensive numerical computations were conducted for different values of the parameters and results are illustrated graphically as well as in tabular form. Selected computations are presented in Figs. 2-12. The correctness of the current numerical method is checked with the results obtained by Mohanty et al. (2015) and is 
shown in table 2. Thus, it is seen from table 2 that the numerical results are in close agreement with those published previously.

\subsection{Effect of suction $\left(\mathrm{V}_{0}\right)$}

Fig.2 illustrates the variation of velocity profiles for various values of suction parameter $\left(V_{0}\right)$. It is observed from this figure that the velocity decreases with increase in the value of the suction parameter. This is because of the reality that the momentum boundary layer thickness depreciates with increment in $\left(V_{0}\right)$. Further, it is seen that the velocity decreases with increase in $\eta$ till it satisfies the boundary condition at $\eta \rightarrow \infty$. Fig. 3 illustrates the effect of $\left(V_{0}\right)$ on micro-rotation profiles. Since there is no buoyancy term occurs in the angular momentum equation, then there exist strong coupling between translational velocity and micro-rotation fields. The linear momentum equation (11) also contains the micro-rotation term, which further gives coupling between velocity and angular velocity fields. All the profiles have different values at $\eta=0$ because of the initial condition $h(0)=-s f^{\prime \prime}(0)$ which is always non-zero since $s=0.5$ and $f^{\prime \prime}(0) \neq 0$. The effect of $\left(V_{0}\right)$ on temperature profiles $(\theta)$ is shown in Fig.4. It is seen from this figure that temperature profiles decreases with increase in the values of $\left(V_{0}\right)$. This is due to the fact that the presence of wall suction has the tendency to reduce the thermal boundary layer thickness which results the reduction in the temperature profiles. Fig.5 depicts the changes in concentration profiles for different values of suction parameter $\left(V_{0}\right)$. This figure indicates that increase in suction parameter $\left(V_{0}\right)$ decreases concentration profiles and is because of the imposition of suction/injection parameter into the flow region depreciate the concentration of the species in the solutal boundary layer. Therefore, From the above Figs. 2 - 5 we observed that the imposition of wall fluid suction $\left(V_{0}>0\right)$ in the present problem of flow has the effect of depreciating the velocity, micro-rotation, temperature and concentration boundary layer thicknesses at every finite value of $\eta$. The deceleration in all profiles with the higher values of suction parameter $\left(V_{0}>0\right)$ is from the reality that suction is taken away the warm fluid from the fluid region.

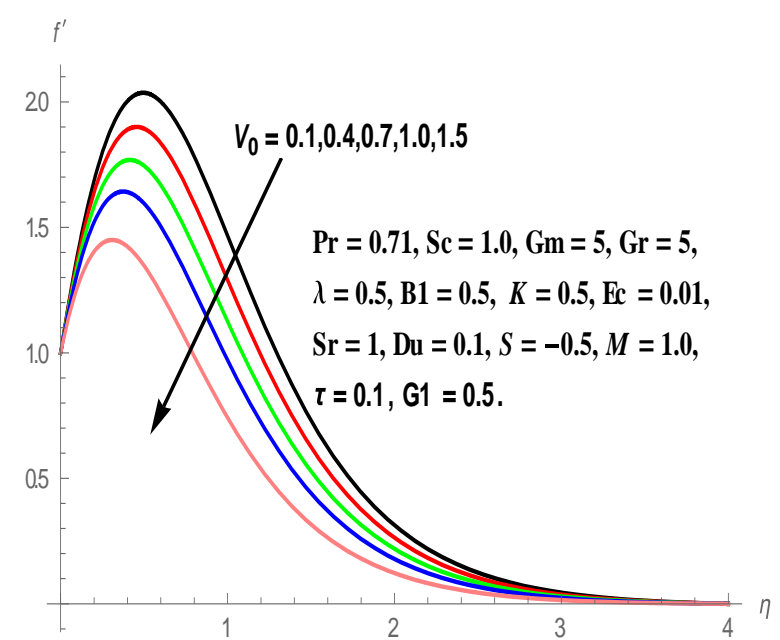

Fig. 2: Effect of $\mathrm{V}_{0}$ on velocity profile.

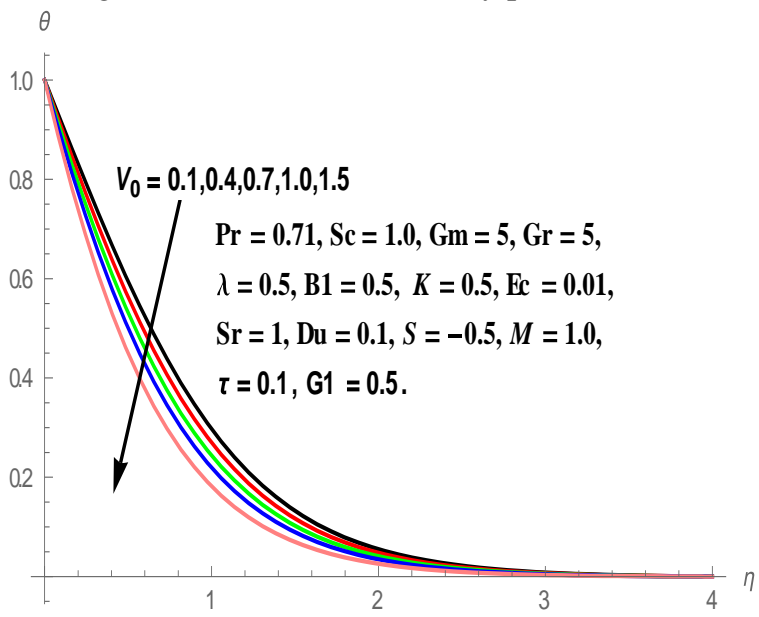

Fig. 4: Effect of $\mathrm{V}_{0}$ on temperature profile.

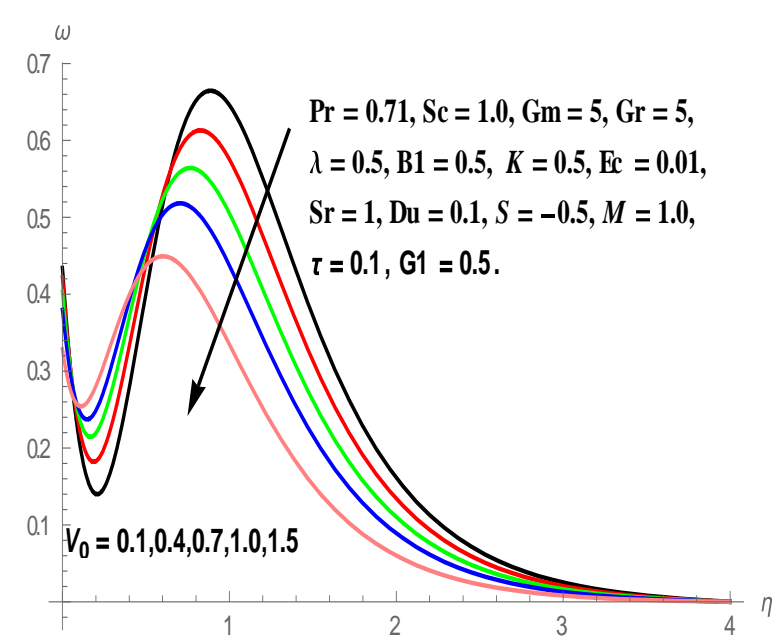

Fig. 3: Effect of $\mathrm{V}_{0}$ on micro-rotation profile

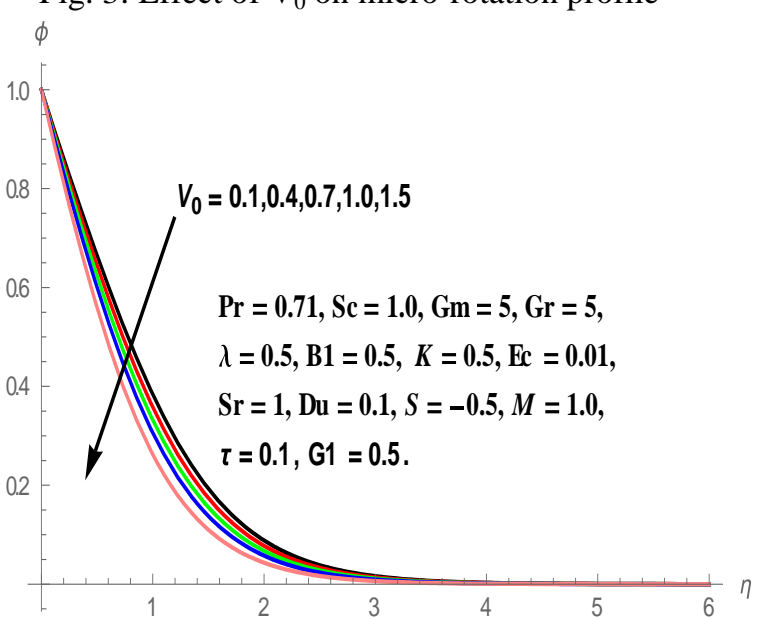

Fig. 5: Effect of $\mathrm{V}_{0}$ on concentration profile 
The variation of local skin-friction co-efficient $\left(f^{\prime \prime}(0)\right)$, local Nusselt number $\left(-\theta^{\prime}(0)\right)$, and local Sherwood number $\left(-\varphi^{\prime}(0)\right)$ for different values of suction parameter $\left(V_{0}\right)$ is presented in table 3 . It is seen from table that the local skin-friction co-efficient depreciates whereas the dimensionless heat and mass transfer rates elevates with increase in suction parameter $\left(V_{0}\right)$.

\subsection{Effect of magnetic parameter (M)}

The influence of magnetic field parameter $(M)$ on velocity, temperature and concentration profiles in the boundary layer is depicted in Figs. 6 - 8. It is noticed from these figures that the hydrodynamic boundary layer thickness depreciates, whereas the thermal boundary layer thickness as well as the solutal boundary layer thickness enhances with the higher values of $(M)$. This is because of the reality that, the presence of magnetic field in an electrically conducting fluid produces a force called Lorentz force, this force acts against the flow direction causes the depreciation in velocity profiles (fig. 6), and at the same time, to overcome the drag force imposed by the Lorentzian retardation the fluid has to perform extra work; this supplementary work can be converted into thermal energy which increases the thickness of thermal and solutal boundary layers in the fluid region (figs. $7 \& 8$ ).

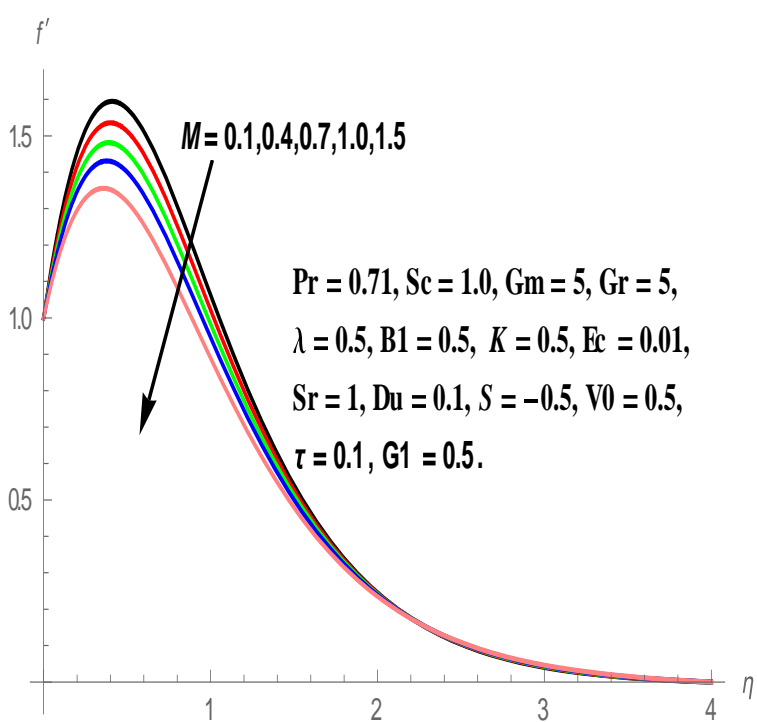

Fig. 6: Effect of $M$ on velocity profile.

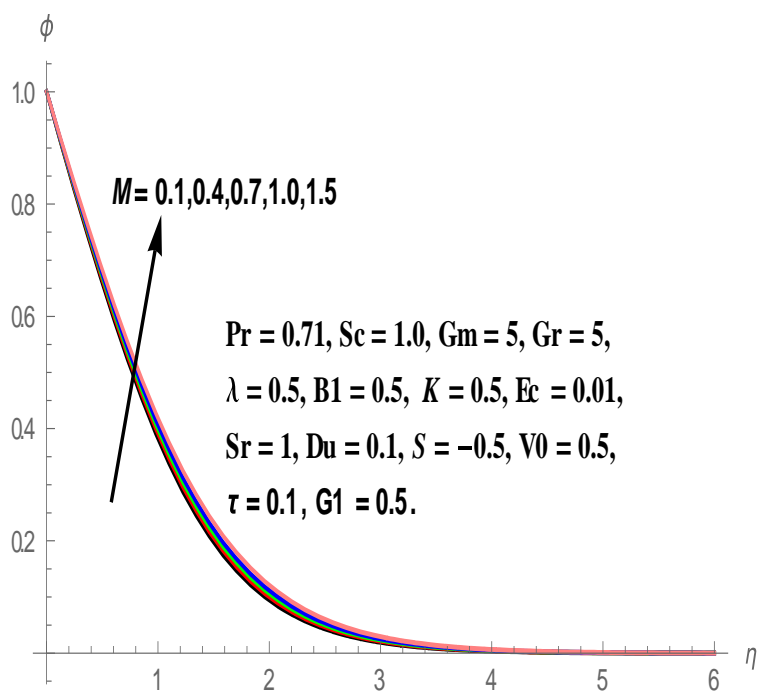

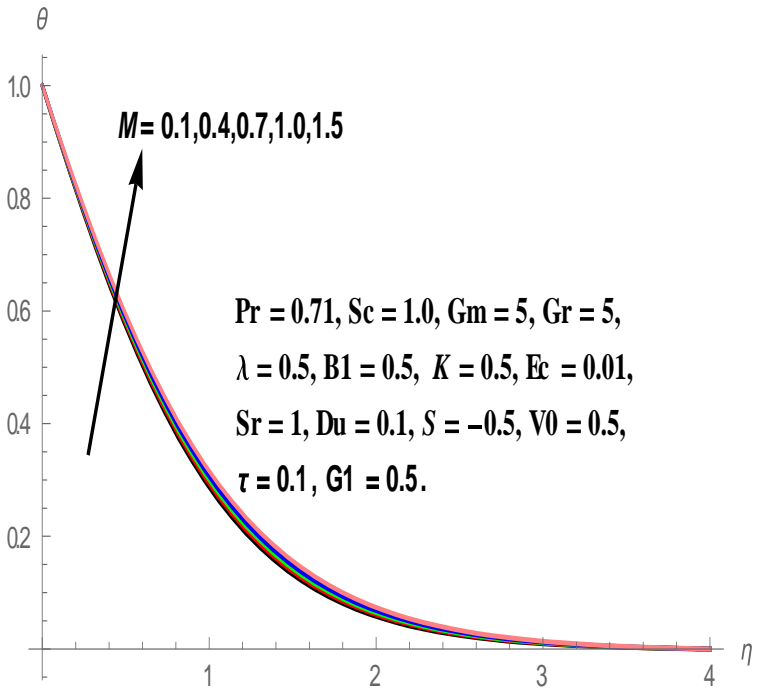

Fig. 7: Effect of $M$ on temperature profile.

Fig. 8: Effect of $M$ on concentration profile

The sway of magnetic parameter $(M)$ on skin-friction coefficient, Nusselt number and Sherwood number is also presented in table 3. It is clear from this table that skin-friction coefficient, dimensionless heat and mass transfer rates are all decelerates with the higher values of magnetic parameter $(M)$. 


\section{$5.3 \quad$ Effect of Soret and Dufour parameters $(\mathrm{Sr} \& \mathrm{Du})$}

The combined impact of $(S r)$ and $(D u)$ on temperature and concentration profiles is depicted in Figs. 9-10. In these graphs $(S r)$ and $(D u)$ values are taken as $S r=0.5,0.8,1.1,1.4,1.8$ and $D u=0.5,0.3,0.2,0.15,0.1$. It is clearly observed from these graphs that the temperature distributions decreases whereas concentration profiles increases at all points in the flow field with the increasing values of Soret number $(S r)$ and the decreasing values of Dufour parameter $(D u)$. This is because of the fact that the diffusive species with higher values of Soret parameter $(\mathrm{Sr})$ has the tendency of increasing concentration profiles whereas thermal species with lower Dufour parameter $(D u)$ values has the tendency of depreciating temperature profiles in the flow field. Thus, it is concluded from Figs. 9 - 10 that the temperature and concentration distributions are more influenced with the values of Soret and Dufour parameters.

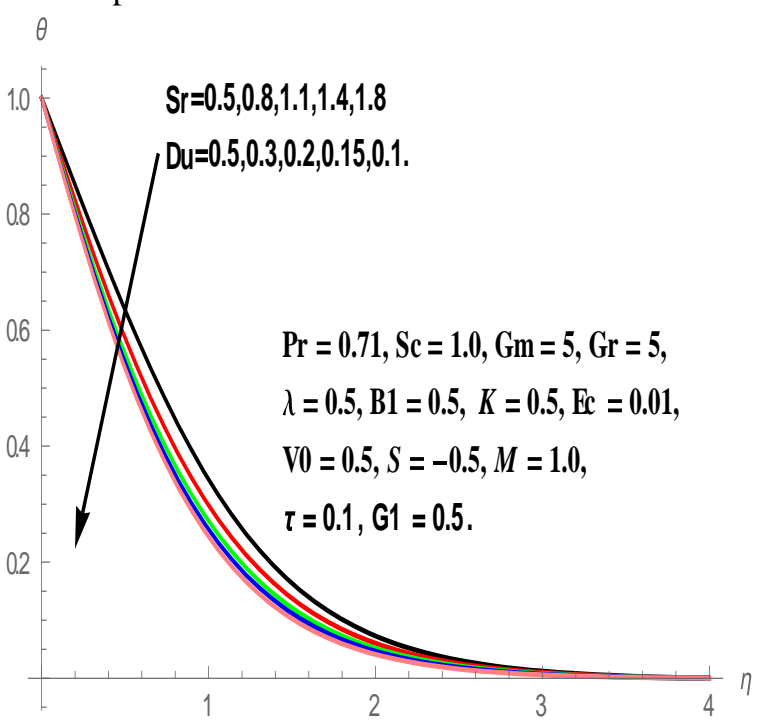

Fig.9: Effect of $S r \& D u$ on temperature profile

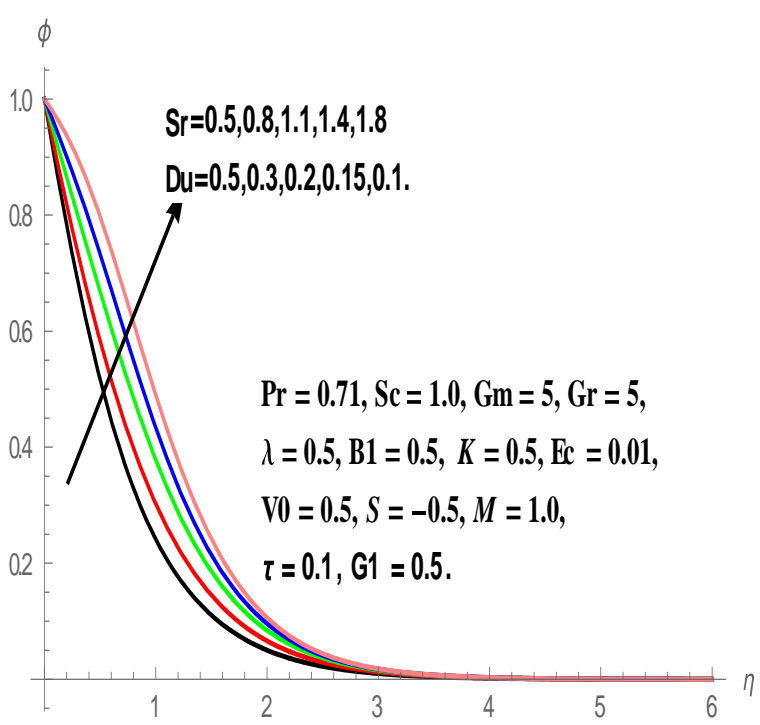

Fig.10: Effect of $S r \& D u$ on concentration profile

The combined influence of $(S r)$ and $(D u)$ on skin-friction coefficient, Nusselt number and Sherwood number is also reported in table 3. It is noticed that $f^{\prime \prime}(0)$ and the dimensionless heat transfer rates are both improved whereas the dimentionless mass transfer rates falls with the increasing values of $(\mathrm{Sr})$ and decreasing values of $(D u)$.

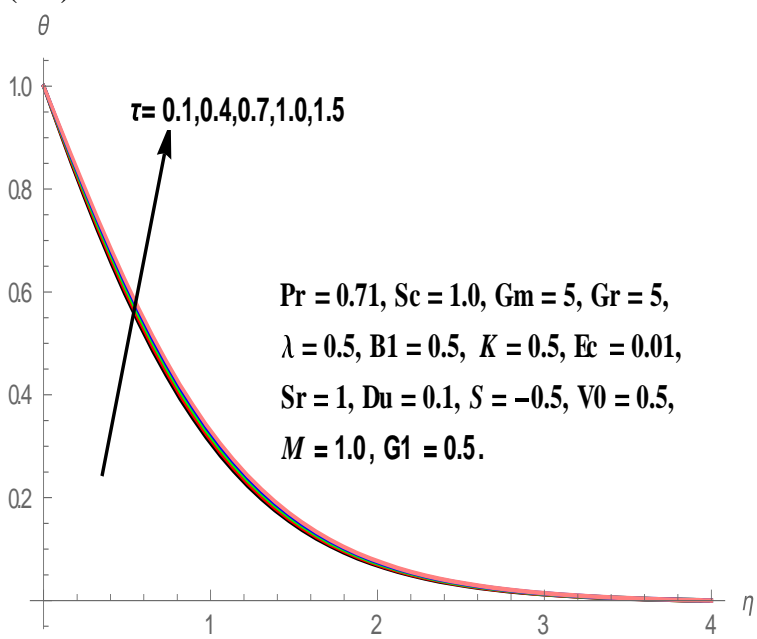

Fig. 11: Effect of $\tau$ on temperature profile

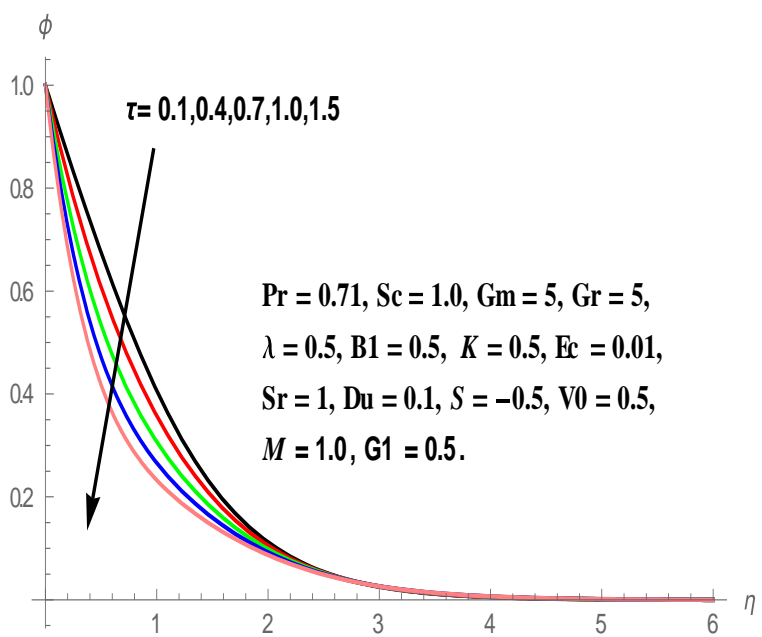

Fig. 12: Effect of $\tau$ on concentration profile

\section{$5.4 \quad$ Effect of thermophoretic parameter $(\tau)$}

The variation in temperature and concentration distributions for various values of thermophoretic parameter $\tau$ is depicted in Figs.11 and 12. It is seen from fig. 11 that the thickness of the thermal boundary layer increases in flow region with higher values of thermophoretic parameter $(\tau)$. This is because of the fact that the particles near the hot surface create a thermophoretic force causes the increment in the thermal boundary layer thickness. 
P. S. Reddy, A. J. Chamkha/ Journal of Naval Architecture and Marine Engineering 13(2015) 39-50 However, the thickness of solutal boundary layers depreciates in the entire flow region with increase in the values of thermophoretic parameter $(\tau)$.

It is evident from table 3 that the skin-friction co-efficient and Nusselt number depreciates whereas Sherwood number enhances with the increasing values of thermophoresis parameter $(\tau)$.

\section{Conclusion}

In the present analysis we have investigated the combined influence of suction and thermophoresis on the mixed convective heat and mass transfer boundary layer flow of a micropolar fluid through porous medium over a stretching sheet in the presence of Thermo-Diffusion and Diffusion-Thermo effects. Similarity transformation is used to transform the resulting partial differential equations into the set of highly non-linear ordinary differential equations and are solved numerically by using Finite element method. The most significant findings of the present study are as follows.

- The velocity, micro-rotation, temperature and concentration profiles are highly influenced with different values of magnetic parameter $(M)$. Velocity profiles impedes because of the Laurentz force, whereas the thickness of thermal and concentration boundary layers are both elevates with higher values of $M$.

- The thickness of hydrodynamic, microrotation, thermal and solutal boundary layers is reduced with the higher values of suction parameter $\left(V_{0}\right)$. This is because of the fact that suction is taken away the warm fluid from the fluid region.

- The thermal boundary layer thickness is improved with the increasing values of thermophoretic parameter $(\tau)$. This is because of the fact that thermophoresis acts against temperature gradient, so that, the particles move from the region of higher temperature to the region of lower temperature.

- The rates of non-dimensionless temperature decreases with an increase in the values of Soret number $S r$.

- The rate of non-dimensionless concentration improves with increasing values of Soret parameter $(S r)$.

- The Nusselt number increases with the decreasing values of Dufour number $(\mathrm{Du})$.

Table 1:Grid-invariance test for velocity distribution $\left(f^{\prime}\right)$, temperature distribution $(\theta)$ and concentration distribution $(\varphi)$, for $\lambda=0.5, \mathrm{G}_{1}=0.5, \mathrm{Ec}=0.01, \mathrm{~B}_{1}=0.5, \mathrm{M}=1.0, \tau=0.1, \mathrm{Pr}=0.71, \mathrm{Sc}=0.22, \mathrm{~K}=0.5, \mathrm{~V} 0$ $=0.5, \mathrm{Gm}=0.1, \mathrm{Gr}=1.0, \mathrm{Sr}=1.0, \mathrm{Du}=0.1$.

\begin{tabular}{|c|c|c|c|c|c|c|c|c|c|c|c|c|}
\hline \multirow[t]{3}{*}{$\eta$} & \multicolumn{4}{|c|}{$f$} & \multicolumn{4}{|c|}{$\theta$} & \multicolumn{4}{|c|}{$\varphi$} \\
\hline & \multicolumn{12}{|c|}{ Step size $(h)$} \\
\hline & 0.04 & 0.02 & 0.01 & 0.005 & 0.04 & 0.02 & 0.01 & 0.005 & 0.04 & 0.02 & 0.01 & 0.005 \\
\hline 0.0 & 1.0000 & 1.0000 & 1.0000 & 1.0000 & 1.0000 & 1.0000 & 1.0000 & 1.0000 & 1.0000 & 1.0000 & 1.0000 & 1.0000 \\
\hline 1.0 & 1.4364 & 1.2563 & 1.1157 & 1.1154 & 0.3174 & 0.2849 & 0.2446 & 0.2445 & 0.3874 & 0.3457 & 0.3289 & 0.3286 \\
\hline 2.0 & 0.3172 & 0.2965 & 0.1874 & 0.1873 & 0.0532 & 0.0321 & 0.0318 & 0.0317 & 0.0965 & 0.0824 & 0.0816 & 0.0814 \\
\hline 3.0 & 0.0534 & 0.0425 & 0.0264 & 0.02631 & 0.0056 & 0.0041 & 0.0011 & 0.0010 & 0.0156 & 0.0098 & 0.0084 & 0.0083 \\
\hline 4.0 & 0.0000 & 0.0000 & 0.0000 & 0.0000 & 0.0000 & 0.0000 & 0.0000 & 0.0000 & 0.0054 & 0.0041 & 0.0034 & 0.0032 \\
\hline 5.0 & ---- & ---- & ---- & ---- & ---- & ---- & ---- & ---- & 0.0005 & 0.0003 & 0.0002 & 0.0001 \\
\hline 6.0 & ---- & ---- & ---- & ---- & ---- & ---- & ---- & ---- & 0.0000 & 0.0000 & 0.0000 & 0.0000 \\
\hline
\end{tabular}

Table 2:Comparison of local Skin-friction, Nusselt number and Sherwood number with the existing results, when $S r=0, D u=0, \mathrm{G} 1=0.5, V o=0, B 1=0.1, \tau=0, \lambda=0.5$.

\begin{tabular}{|c|c|c|c|c|c|c|c|c|c|c|c|c|}
\hline \multicolumn{7}{|c|}{ Parameter } & \multicolumn{2}{|c|}{$C_{f}$} & \multicolumn{2}{|c|}{$N u_{x}$} & \multicolumn{2}{|c|}{$S h_{x}$} \\
\hline $\mathrm{Gr}$ & Gc & $\operatorname{Pr}$ & $\mathrm{Sc}$ & $\mathrm{M}$ & K & Ec & $\begin{array}{l}\text { Mohanty et } \\
\text { al. (2015) }\end{array}$ & $\begin{array}{l}\text { Present } \\
\text { Study }\end{array}$ & $\begin{array}{l}\text { Mohanty et } \\
\text { al. (2015) }\end{array}$ & $\begin{array}{l}\text { Present } \\
\text { Study }\end{array}$ & $\begin{array}{l}\text { Mohanty et } \\
\text { al. (2015) }\end{array}$ & $\begin{array}{l}\text { Present } \\
\text { Study }\end{array}$ \\
\hline 0.0 & 0.0 & 0.72 & 0.00 & 0 & 100 & 0.00 & -0.81861 & -0.81873 & 0.85604 & 0.85619 & 0.16666 & 0.16671 \\
\hline 0.1 & 0.0 & 0.72 & 0.00 & 0 & 100 & 0.00 & -0.78025 & -0.78019 & 0.86514 & 0.86526 & 0.42252 & 0.42263 \\
\hline 0.1 & 0.0 & 0.72 & 0.22 & 0 & 100 & 0.00 & -0.78025 & -0.78019 & 0.86514 & 0.86526 & 0.42252 & 0.42263 \\
\hline 0.1 & 0.0 & 0.72 & 0.22 & 1 & 100 & 0.00 & -1.11781 & -1.11776 & 0.78477 & 0.78482 & 0.37866 & 0.37872 \\
\hline 0.1 & 0.0 & 0.72 & 0.22 & 1 & 0.5 & 0.01 & -1.59782 & -1.59773 & 0.68391 & 0.68386 & 0.33393 & 0.33385 \\
\hline 0.1 & 0.0 & 0.72 & 0.22 & 1 & 100 & 0.01 & -1.11775 & -1.11766 & 0.77999 & 0.77981 & 0.37867 & 0.37874 \\
\hline 0.1 & 0.0 & 0.72 & 0.22 & 1 & 0.5 & 0.01 & -1.59777 & -1.59782 & 0.67651 & 0.67646 & 0.33394 & 0.33398 \\
\hline 0.1 & 0.1 & 7.00 & 0.22 & 1 & 100 & 0.01 & -1.09307 & -1.09311 & 3.02046 & 3.02052 & 0.38361 & 0.38370 \\
\hline 0.5 & 0.1 & 0.72 & 0.22 & 1 & 100 & 0.01 & -0.93986 & -0.93991 & 0.82922 & 0.82931 & 0.40495 & 0.40486 \\
\hline 0.1 & 0.1 & 0.72 & 0.22 & 1 & 100 & 0.01 & -1.06792 & -1.06798 & 0.79858 & 0.79863 & 0.38891 & 0.38896 \\
\hline 0.5 & 0.1 & 0.72 & 0.22 & 1 & 0.5 & 0.01 & -1.44651 & -1.44667 & 0.72576 & 0.72569 & 0.35583 & 0.35591 \\
\hline
\end{tabular}


Table 3: The local Skin-friction, Nusselt number and Sherwood number for different values of the important parameters.

\begin{tabular}{|c|c|c|c|c|c|c|c|}
\hline$V_{0}$ & $\mathrm{M}$ & $\mathrm{Sr}$ & $\mathrm{Du}$ & $\tau$ & $f^{\prime \prime}(0)$ & $-\theta^{\prime}(0)$ & $-\varphi^{\prime}(0)$ \\
\hline 0.1 & 1.0 & 1.0 & 0.1 & 0.1 & 4.341664 & 0.840417 & 0.713787 \\
0.4 & 1.0 & 1.0 & 0.1 & 0.1 & 4.217637 & 0.957082 & 0.766098 \\
0.7 & 1.0 & 1.0 & 0.1 & 0.1 & 4.035646 & 1.081757 & 0.820302 \\
1.0 & 1.0 & 1.0 & 0.1 & 0.1 & 3.797455 & 1.214205 & 0.877212 \\
0.5 & 0.1 & 1.0 & 0.1 & 0.1 & 3.181322 & 0.956905 & 0.727742 \\
0.5 & 0.4 & 1.0 & 0.1 & 0.1 & 2.951362 & 0.946389 & 0.714376 \\
0.5 & 0.7 & 1.0 & 0.1 & 0.1 & 2.735015 & 0.936191 & 0.701742 \\
0.5 & 1.0 & 1.0 & 0.1 & 0.1 & 2.531069 & 0.926322 & 0.689786 \\
0.5 & 1.0 & 0.5 & 0.5 & 0.1 & 4.073383 & 0.743463 & 1.165773 \\
0.5 & 1.0 & 0.8 & 0.3 & 0.1 & 4.130123 & 0.897880 & 0.960502 \\
0.5 & 1.0 & 1.1 & 0.2 & 0.1 & 4.304030 & 0.985056 & 0.688923 \\
0.5 & 1.0 & 1.4 & 0.1 & 0.1 & 4.304302 & 1.025185 & 0.482014 \\
0.5 & 1.0 & 1.0 & 0.1 & 0.1 & 2.531069 & 0.926322 & 0.689786 \\
0.5 & 1.0 & 1.0 & 0.1 & 0.4 & 2.403028 & 0.899578 & 0.967457 \\
0.5 & 1.0 & 1.0 & 0.1 & 0.7 & 2.262505 & 0.867547 & 1.307852 \\
0.5 & 1.0 & 1.0 & 0.1 & 1.0 & 2.140653 & 0.837243 & 1.637596 \\
\hline
\end{tabular}

\section{Reference}

Abd El-Aziz, M. (2013): Mixed convection flow of a micropolar fluid from an unsteady stretching surface with viscous dissipation, Journal of the Egyptian Mathematical Society, Vol. 21, pp. 385-394.

Alam, M.S., Ferdows,M., Ota, M. and Maleque, M.A. (2006a): Dufour and Soret effects on steady free convection and mass transfer flow past a semi-infinite vertical porous plate in a porous medium,International Journal of Applied Mechanics and Engineering, Vol. 11, pp. 535-545.

Alam, M.S. and Rahman, M.M. (2006b): Dufour and Soret effects on mixed convection flow past a vertical porous flat plate with variable suction, Non Linear Analysis; Modelling and Control, Vol. 11, pp. 3-12.

Anwar Bég, O., Takhar, H.S., Bhargava, R., Rawat, S. and Prasad, V.R. (2008): Numerical study of heat transfer of a third grade viscoelastic fluid in non-Darcian porous media with thermo physical effects, Physica Scripta, Vol.77, pp. 1-11.

Ariman, T., Turk, M.A. and Sylvester, N.D. (1973): Microcontinuum fluid mechanics-review, International Journal of Engineering Scence, Vol.11, pp. 905-930.

Ariman, T., Turk, M.A. and Sylvester, N.D. (1974): Application of microcontinuum fluid mechanics, International Journal of Engineering Scence, Vol.12, pp. 273-293.

Malga, B. S. and Kishan, N. (2014): Finite element analysis for unsteady MHD heat and mass transfer free convection flow of polar fluids past a vertical moving porous plate in a porous medium with heat generation and thermal diffusion, Journal of Naval Architecture and Marine Engineering, vol. 11, no. 1, pp. 69-82. http://dx.doi.org/10.3329/jname.v11i1.12844

Bhargava, R., Kumar, L. and Takhar, H.S. (2003): Finite element solution of mixed convection microploar fluid driven by a porous stretching sheet, International Journal of Engineering Scence, Vol. 41, pp. 2161-2178.

Bhargava, R., Sharma, R and Bég, O.A. (2009): Oscillatory chemically-reacting MHD free convection heat and mass transfer in a porous medium with Soret and Dufour effects, finite element modeling, International Journal Applied Mathematics and Mechanics, Vol, 5, pp. 15-37.

Chamka, A.J., Takhar, H.S. and Soundalgekar, V.N. (2011a): Radiation effect on free convection flow past a semi-infinite vertical plate with mass transfer, Chemical Engineering journal, Vol. 84, pp. 335-342.

Chamkha, A.J. and Pop, I. (2004): Effect of thermophoresis particle deposition in free convection boundary layer from a vertical flat plate embedded in a porous medium, International Communications in Heat and Mass Transfer, Vol. 31, pp. 421-430.

Chamkha, A.J., Mohammad, R.A. and Ahmad, E. (2010): Unsteady MHD natural convection from a heated vertical porous plate in a micropolar fluid with Joule heating, chemical reaction and radiation effects, Meccanica, 2010, DOI 10.1007/s11012-010-9321-0.

Chamkha, A. J., EL-Kabeir, S. M. M. and Rashad, A. M. (2013): Coupled heat and mass transfer by MHD natural convection of micropolar fluid about a truncated cone in the presence of radiation and chemical reaction, 
P. S. Reddy, A. J. Chamkha/ Journal of Naval Architecture and Marine Engineering 13(2015) 39-50 Journal of Naval Architecture and Marine Engineering, vol. 10, no. 2, pp. $157-168$. http://dx.doi.org/10.3329/jname.v10.i2.15898

Chamkha, A.J., El-Amin, M.F. and Aly, A.M. (2011b): Unsteady double-diffusive natural convective MHD flow along a vertical cylinder in the presence of chemical reaction, thermal radiation and Soret and Dufour effects, Journal of Naval Architecture and Marine Engineering, DOI: 10.3329/jname.v8i1.7250.

Chapman, S. and Cowling, T.G. (1952): The Mathematical Theory of Non-Uniform Gases, Cambridge University Press, Cambridge, UK.

Damseh, R. A., Al-Odat, M.Q., Chamkha, A.J. and Shannak, B. A. (2009): Combined effect of heat generation or absorption and first-order chemical reaction on micropolar fluid flows over a uniformly stretched permeable surface, International Journal of Thermal Science, Vol. 48, pp. 1658-1663.

Pal, D. and Mondal, H. (2011): MHD non-Darcian mixed convection heat and mass transfer over a non-linear stretching sheet with Soret and Dufour effects and chemical reaction, International communications in heat and mass transfer, Vol.38, pp. 463-467.

Eringen, A.C. (1996): Theory of micropolar fluids, Journal Mathematics and Mechanics, Vol.16, pp.1-18.

Eringen, A.C. (2001): Microcontinuum field theories II, fluent media. Springer, New York.

Goren, S.L. (1977): Thermophoresis of aerosol particles in the laminar boundary layer on a flat

plate, Journal of Colloid Interface Science, Vol. 61, pp. 77-85.

Hirshfelder, J.O., Curtis, C.F. and Bird, R.B. (1954): Molecular Theory of Gases and Liquids, Welly New York. Ibrahim, F.S., Elaiw, A.M. and Bakr, A.A. (2008): Influence of viscous dissipation and radiation on unsteady MHD mixed convection flow of micropolar fluids, Applied Mathematics \& Information Science, Vol. 2, pp. $143-162$.

Lukaszewicz, G. (1999): Micropolar fluids: theory and application, Birkhäuser Basel.

Mahmood, R., Nadeem, S. and Akber, N.S. (2013): Non-orthogonal stagnation point flow of a micropolar second grade fluid towards a stretching surface with heat transfer, Journal of the Taiwan Institute of Chemical Engineering, Vol. 44, pp. 586-595.

Makinde, O.D. (2011): On MHD mixed convection with soret and dufour effects past a vertical plate embedded in a porous medium, Latin American Applied Research, Vol. 41, pp. 63-68.

Mohanty, B., Mishra, S.R. and Pattanayak, H.B. (2015): Numerical investigation on heat and mass transfer effect of micropolar fluid over a stretching sheet through porous media, Alexandria Engineering Journal, Vol. 54, pp. 223-232.

Partha, M.K. (2008): Thermophoresis particle deposition in a non-Darcy porous medium under the influence of Soret, Dufour effects, Heat and Mass Transfer, Vol. 44, pp. 969-977.

Partha, M.K. (2009): Suction/injection effects on thermophoresis particle deposition in a non-Darcy porous medium under the influence of Soret and Dufour effects, International Journal of Heat and Mass Transfer, Vol. 52, pp. 1971-1979.

Prathap Kumar, J., Umavathi, J.C., Chamkha, A.J. and Pop, I. (2010): Fully developed free convective flow of micropolar and viscous fluids in a vertical channel, Applied Mathematics Modelling, Vol.34, pp. 1175-1186.

Raju, M.C. andVarma, S.V.K. (2014): Soret effects due to natural convection in a non-Newtonian fluid flow in porous medium with heat and mass transfer, Journal of Naval Architecture and Marine Engineering, vol. 11, no. 2, pp. 147-156. http://dx.doi.org/10.3329/jname.v11i2.17563

Rana, P., and R. Bhargava (2012): Flow and heat transfer of a Nano fluid over a nonlinearly stretching sheet: a numerical study, Communications in Nonlinear Science and Numerical Simulation, Vol.17,pp. 212-226.

Reddy, P.S., and Rao. V.P. (2012): Thermo-diffusion and diffusion -thermo effects on convective heat and mass transfer through a porous medium in a circular cylindrical annulus with quadratic density temperature variation - Finite element study,Journal of Applied Fluid Mechanics, Vol, 5, pp. 139-144.

Rosali, H., Ishak, A. and Pop, I. (2012): Micropolar fluid flow towards a stretching/shrinking sheet in a porous medium with suction, International Communications in Heat and Mass Transfer, Vol. 39, pp. 826-829.

Seddeek, M.A. (2005): Finite element method for the effects of chemical reaction, variable viscosity, thermophoresis and heat generation/absorption on a boundary layer hydromagnetic flow with heat and mass transfer over a heat surface, Acta Mechanica, Vol. 177, pp. 1-18.

Yacos, N.A., Ishak, A. and Pop, I. (2011): Melting heat transfer in boundary layer stagnation-point flow towards a stretching/shrinking sheet in a micropolar fluid, Computers \& Fluids, Vol. 47, pp. 16-21. 